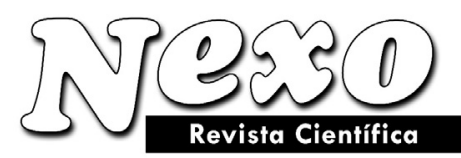

\title{
Instrumental para la valoración ambiental del medio construido de los asentamientos rurales
}

\author{
H. Hernández* \\ Dirección de Desarrollo Educativo, Universidad Nacional de Ingeniería (UNI) \\ PO Box 5595, Managua, Nicaragua \\ e-mail: hernandezheim@gmail.com
}

(Recibido/received: 28-Jul-2009; aceptado/accepted: 17-Agosto-2009

\begin{abstract}
RESUMEN
El instrumental contiene un sistema de indicadores, obtenidos de la base del estudio de conceptos y criterios relevantes, y de la caracterización de los asentamientos rurales, que incluye parámetros para evaluar la calidad ambiental del medio construido de los asentamientos rurales. El instrumental diseñado tiene implícito el enfoque sistémico e integral, en aras de alcanzar la sustentabilidad local y fue validado en cinco asentamientos rurales del municipio de Masaya, Nicaragua. El sistema de indicadores se obtuvo de un conjunto de 40 variables significativas, las cuales se interrelacionaron gráficamente tomando en cuenta la teoría de sistemas, $\mathrm{y}$, mediante el proceso por asociación y significado, se obtuvieron 15 indicadores específicos y seis indicadores generales, con su correspondiente sistema categorial. Los resultados de la validación son visualizados en histogramas y matrices. En conclusión, el instrumental permite medir y valorar la calidad ambiental de los asentamientos, evidenciando las carencias y las potencialidades para mejorar las condiciones de vida de sus habitantes. Induce también a orientar el desarrollo integral del asentamiento, así como su desarrollo dentro del sistema territorial.
\end{abstract}

Palabras claves: calidad ambiental; asentamientos rurales; indicadores; valoración ambiental.

\begin{abstract}
The instrumental contains a system of indicators obtained from a study based on relevant concepts and criteria as well as the characterization of rural settlements. The system of indicators includes parameters which evaluate the environmental factors that constitute the rural settlement. The instrumental design has an implicit systemic and integral focus in order to achieve local sustainability. The instrument has been validated in five rural settlements within the municipality of Masaya, Nicaragua. The system of indicators was obtained from a compilation of the most significant forty variables or components. Taking into account the Theory of Systems and using a process of association, significance and graphical interrelationships fifteen specific indicators were obtained as well as six general indicators with their corresponding system category. The results of the validation are shown using histograms and matrices. In conclusion, the instrumental allows measurement and assessment of the environmental quality of settlements. It also demonstrates limitations as well as potential for improvement in living conditions for residents. The instrument can also act as a guide for integral development within rural settlements as well as the wider regions.
\end{abstract}

Keyword: environmental quality; indicators; rural settlements; environmental assessment.

\footnotetext{
*Autor para la correspondencia
} 


\section{INTRODUCCIÓN}

En la actualidad muchos de los asentamientos rurales carecen de sistemas de infraestructura, de servicios y de equipamientos que le otorguen adecuadamente el sustento ambiental, debido al criterio de integración con el territorio. En Nicaragua el territorio evidencia una inefectiva planificación territorial con enfoque sistémico, donde el medio construido refleja la calidad de vida de su población. En este sentido, se necesita medir y valorar la calidad de vida o calidad ambiental de los asentamientos rurales, para mejorar las condiciones físicas del medio construido y de sus habitantes.

Se analizaron modelos e instrumentales que tienen como objetivo determinar la calidad de vida mediante indicadores, siendo algunos ejemplos: Organización para la Cooperación y el Desarrollo Económico (OCDE), 1993; Organización Mundial de la Salud (OMS), 1993; United Nations Centre for Human Settlements-HABITAT (UNCHS), 1997, Comisión de Comunidades Europeas, 2002, Observatorios Ambientales Urbanos, 2002. Hasta el momento no existe un instrumento que mida la calidad ambiental de forma integral. Se tienen ejemplos en América Latina con Conesa-Fernández (1994), Pérez (2000), Velásquez (2003) y Gómez (2005), de los cuales, Pérez y Gómez tienen aplicabilidad en los asentamientos rurales, y en relación a una visión sistémica, los ejemplos existentes son Gómez (2005) y Velásquez (2003).

Los instrumentales latinoamericanos son resultantes de un sistema organizacional enmarcado en un contexto espacio-temporal, y por tanto, evidencian qué necesitan medir o evaluar para obtener una calidad ambiental. Para ello, en el caso de Nicaragua, es necesario detectar los componentes o variables significativas inmersas en el medio construido rural.

Para validar el instrumental de evaluación de la calidad ambiental del medio construido de los asentamientos rurales, se conformó un grupo de profesionales con experiencia técnica y práctica, con categorías científicas y/o académicas, que a la vez tuvieran conocimientos del tema, de las normativas y poseyeran un amplio aval de trabajo, con posibilidad de emitir criterios relevantes en torno al instrumental teórico-práctico de evaluación generado. Se agruparon profesionales de las siguientes instituciones: Alcaldía de la ciudad de Masaya, Universidad Nacional de
Ingeniería, Facultad de Arquitectura, tutores del doctorado en Ciencias del Ambiente y del Programa de Estudios Ambientales Urbano Territoriales (PEAUT-UNI).

\section{METODOLOGÍA}

Se investigó la problemática referida a la estructuración de modelos e indicadores de evaluación de calidad ambiental, coincidiendo con Gómez (2005) acerca de la regularidad del uso de los términos para calificar los componentes que conforman el sistema de evaluación. Se hizo una adaptación de la estructura correspondiente al planteo de los modelos analizados en América Latina: Gómez (2005), Pérez (2000) y Velásquez (2003); el primero por poseer una organización fácil de comprensión, categorías cualitativas de evaluación y uso de histogramas y matrices; el segundo por poseer un sistema categorial de indicadores. Ambos modelos son aplicados al escenario rural. El tercer modelo por aplicar la evaluación mediante histogramas y matrices, además de la concepción sistémica planteada.

El instrumental se conforma de un sistema categorial de indicadores generales e indicadores específicos, cuyos componentes más significativos fueron definidos por medio del proceso de interrelaciones. La aplicación de dicho instrumental es teórico-práctico, siendo su estructura la referida a continuación (ver figura 1).

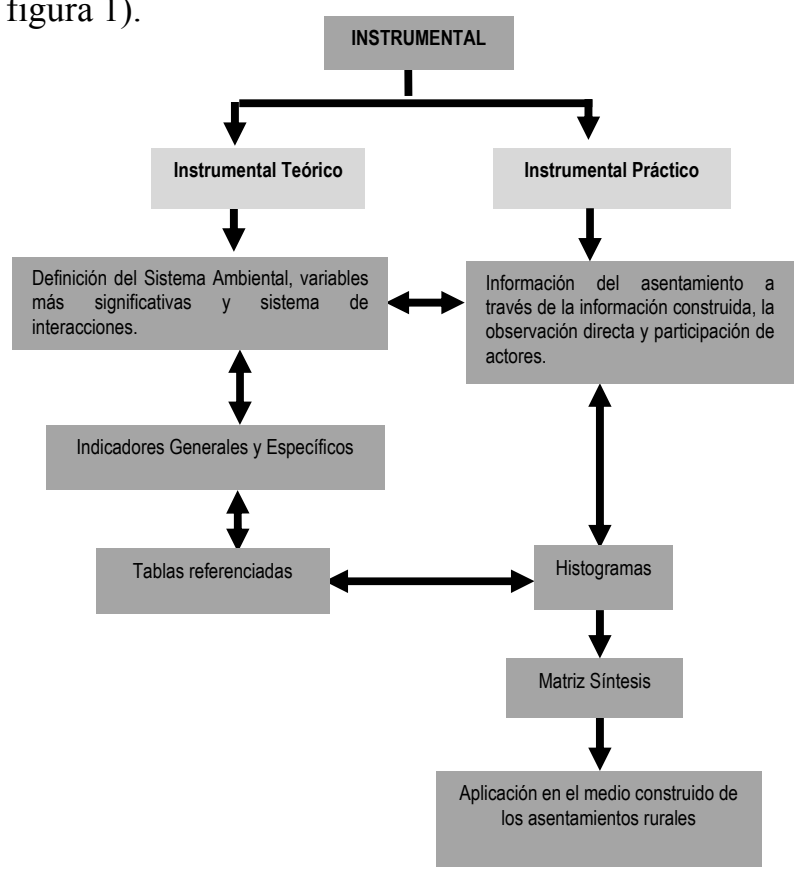

Fig. 1 Estructura del instrumental teórico-práctico. 


\section{H. Hernández}

\section{Sistema categorial del instrumental}

Las categorías asumidas en el instrumental fueron precisadas y adecuadas en esta investigación, aportándose las siguientes definiciones de los componentes que conforman el sistema categorial:

- Sistema de Interacción Ambiental (SI): Es una expresión gráfica en la que se define el número de subsistemas que componen el sistema ambiental a estudiar. Cada subsistema está integrado por las variables que inciden en el mismo, las cuales se interrelacionan, produciendo los indicadores que se utilizarán para medir la calidad ambiental.

- Variables: son los componentes presentes en el sistema ambiental y de los cuales se obtuvieron los indicadores específicos, mediante un sistema de interacción. Estos son atributos del medio, sujetos a un rango, según las condiciones y circunstancias en que se manifiesten.

- Indicadores: son signos, manifestaciones, muestras de algún suceso, acontecimiento o proceso, que ponen en evidencia la magnitud o intensidad de un problema o el grado de impacto alcanzado. Para ello, el indicador debe poseer los siguientes requisitos: validez, confiabilidad, efecto demostrativo y pertinencia. Para la presente investigación, los indicadores establecen la medición cualitativa del medio construido mediante la asignación de parámetros cualitativos.

- Indicadores Específicos (IE): son el resultado del sistema de interacciones entre las variables existentes en el medio construido de los asentamientos rurales.

- Indicadores Generales (IG): Constituyen los elementos generales del sistema ambiental de los asentamientos rurales, declarando la situación del sistema ambiental. Fueron obtenidos mediante la agrupación común entre Indicadores Específicos.

- Escala de Evaluación: Es la medición cuantitativa y cualitativa de los indicadores específicos presentes en el sistema ambiental, cuyos valores determinan la calidad ambiental del medio construido del asentamiento rural. La escala de evaluación se indica en parámetros, que son:

- Parámetros Cualitativos: representan la dimensión que mide cualitativamente el indicador en los asentamientos, establecidos según la escala: Excelente, Bueno, Regular y Malo (Gómez, 2005). En esta investigación se determinó el valor cualitativo de la siguiente manera: Excelente (verde oscuro), Bueno (amarillo), Regular (anaranjado) y Malo (rojo).
- Parámetros Cuantitativos: son los rangos cuantitativos obtenidos de la ponderación de los parámetros cualitativos, teniendo la siguiente escala: Excelente $(4,50-5,00)$, Bueno $(3,80-$ $4,49)$, Regular $(3,00-3,79)$ y Malo $(2,00-$ $2,99)$.

A partir de la definición del sistema categorial, se diseñaron los instrumentales teórico y práctico para la evaluación.

\section{Instrumental teórico de evaluación}

Los indicadores de evaluación se determinaron a partir de la interacción de las variables más significativas del sistema ambiental definido para los asentamientos rurales, así como de los criterios aportados por normativas, especialistas y actores. Se incorporan además, el modo de vida local, el sistema de edificaciones e infraestructuras para el área rural, la accesibilidad del asentamiento y el funcionamiento del mismo.

A continuación se describen los principales pasos para la obtención de los indicadores de evaluación:

- Interacción sistémica de las variables más significativas.

- Obtención de Indicadores Específicos (IE).

- Agrupación de IE en Indicadores Generales (IG) por asociación.

- La cualificación de los IE incide en la de los IG, los cuales dictaminan la evaluación integral del asentamiento generando el Indicador de la Calidad Ambiental.

Los indicadores generales constituidos son:

- Sistema de infraestructuras técnicas: considera la calidad y existencia del sistema de infraestructuras: energía eléctrica y su obtención y distribución por formas tradicionales o alternativas; agua potable, redes telefónicas, drenaje pluvial, evacuación y tratamiento de las aguas negras, tratamiento de desechos sólidos, sistema pluvial y vías de comunicación.

- Características de la vivienda rural: toma en cuenta los elementos formales-espaciales y funcionalesespaciales; obteniendo la calidad de la misma con la consideración de los componentes del modo de vida local.

- Sistema de equipamiento: refleja el sistema de edificaciones del asentamiento y su calidad en el servicio, de acuerdo a la jerarquía de asentamiento 


\section{H. Hernández}

- rural establecido en el Anteproyecto de la Ley General de Ordenamiento y Desarrollo Territorial (2003).

- Percepción social y el asentamiento rural: evalúa la subjetividad social de la percepción y aceptación del asentamiento, incluyendo la relación de la cultura y la legislación con el medio construido.

- El asentamiento rural y su entorno natural: relacionado con la percepción a nivel del medio natural del asentamiento.

- Accesibilidad del asentamiento rural: considera lo relativo al concepto de la accesibilidad al asentamiento, implicando la funcionalidad del mismo, teniendo como reflejo la estructura del asentamiento y la cultura local.

\section{Sistema de interacciones del instrumental teórico}

El Sistema de Interacciones determinó los indicadores significativos, se realiza mediante un gráfico que comprende los tres medios del sistema ambiental para los asentamientos rurales. Esta forma de realizar las interacciones es una adaptación del creado por Valentín-Spangenberg (2000), Velásquez (2003) y Mendoza (2007). Se obtiene el cruce de una variable que tenga relación imprescindible con otras variables de los tres medios en el sistema ambiental, lográndose una interacción articulada, integral y sistémica.

El medio construido se tomó como eje para realizar las interacciones de las 20 variables más significativas, debido a que es la concreción del medio social, compuesto por 11 variables, y que afecta o beneficia al medio natural, compuesto por 9 variables. Como resultado se obtuvieron 15 indicadores específicos, los cuales miden la calidad ambiental de los asentamientos rurales.

En el proceso para obtener los indicadores se visualizó que más de un sistema de interacciones sirvió para obtener un indicador. La agrupación de indicadores específicos concentra un indicador general. Se muestra un ejemplo de la forma de lograr los indicadores a partir de las interacciones. (Ver figura 2). En este caso el Sistema de Interacción SI-1 refleja la relación del Sistema de Redes de Comunicación, es decir, carreteras, andenes, caminos, senderos, puentes, ríos y su sistema de transportación con las siguientes variables: uso de suelo, legislación sentido de equidad, sentido de propiedad, producción local, tradiciones, empleo, instalaciones de producción, dinámica poblacional, morfología del asentamiento, suelo, obtención de la energía paisaje natural, paisaje construido y estructura del asentamiento. Obteniendo así el indicador específico: Accesibilidad del asentamiento rural.

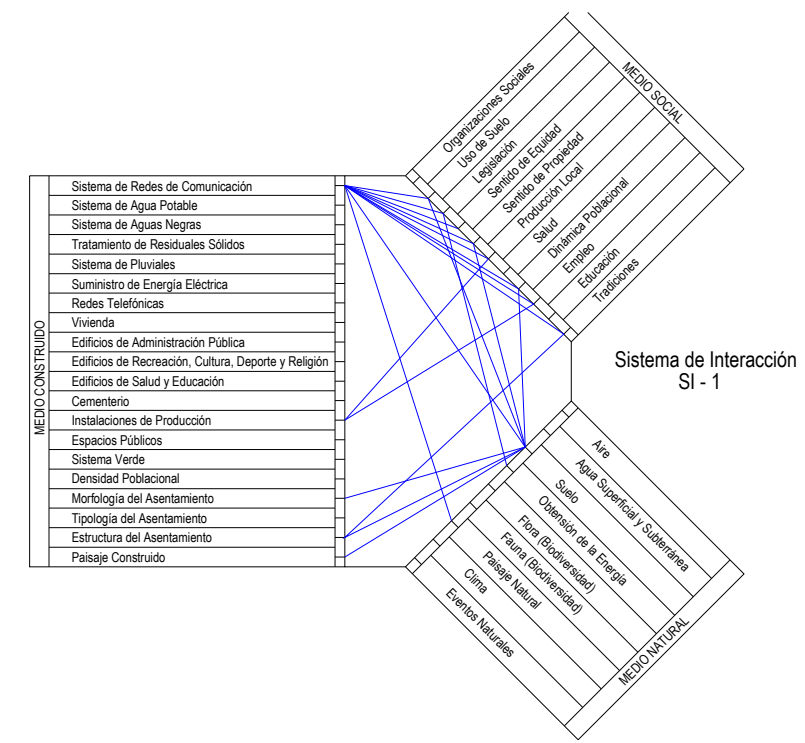

Fig. 2 Interacción entre variables más significantes del sistema ambiental para los asentamientos rurales.

\section{Parámetros de evaluación del instrumental teórico}

Los parámetros de evaluación son parte del instrumental teórico e incorporan la escala de evaluación cuantitativa y cualitativa del sistema de indicadores específicos para la valoración ambiental del medio construido. Parten de una expresión cualitativa, en la cual se utilizó la normativa existente, experiencia de la autora, comité de expertos, modelos estudiados y datos estadísticos regionales y del país.

Para ello se elaboró un conjunto de tablas referenciadas, una para cada indicador. Cada tabla expresa los parámetros diseñados para el instrumental, que parten de una expresión cualitativa del modelo de calidad ambiental con su escala de valores, establecida en: Excelente, Bueno, Regular y Malo.

Estos poseen rangos cuantitativos resultado de la ponderación de los parámetros cualitativos y asociándolos a ellos como: Excelente $(5,00)$, Bueno $(4,00)$, Regular $(3,00)$ y Malo $(2,00)$; y el modo de obtener el cálculo para la obtención del valor cuantitativo con la correspondiente unidad de medida del indicador. (ver tabla 1) 


\section{H. Hernández}

Tabla 1 Parámetros de evaluación de la calidad ambiental.

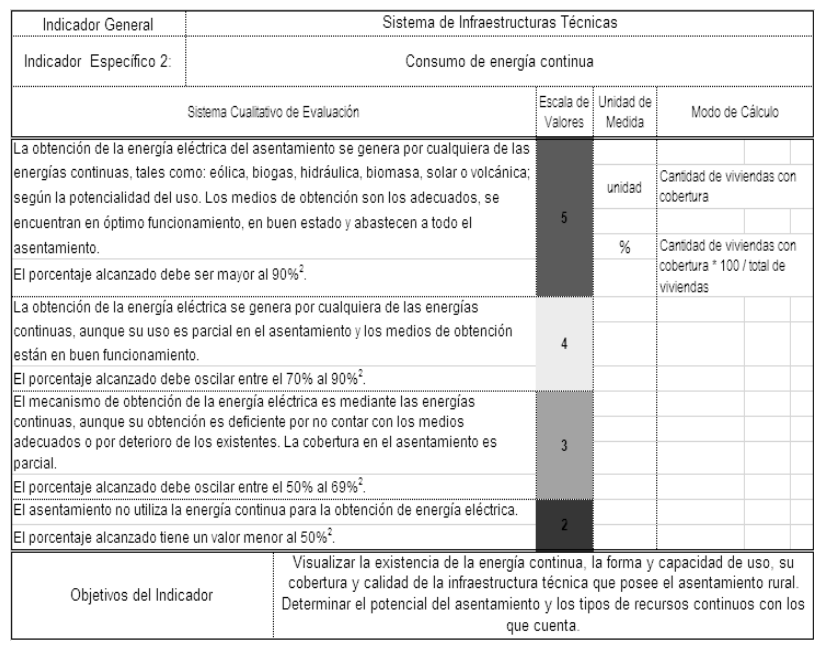

Instrumental práctico de evaluación

Con el instrumental teórico conformado por el sistema de indicadores, se elaboró un procedimiento avalado por procesos matemáticos que posibilitan una aplicación objetiva del mismo y que representa el instrumental práctico conformado por histogramas y matrices que grafican los resultados de las tablas referenciadas. Con la elaboración de histogramas se obtienen los valores cuantitativos y cualitativos de todos los indicadores específicos. La matriz refleja la evaluación total de cada indicador general del asentamiento.

Se asume la realización de histogramas por ser gráficas estadísticas que permiten expresar valores agrupados en intervalos. Estas gráficas han sido utilizadas en los modelos de Contreras-Cordero (1994); Velásquez (2003) y en Gómez (2005). El histograma se expresa como un instrumento cualitativo y evaluativo en esta investigación.

La matriz es la síntesis de la representación gráfica de la evaluación final del comportamiento de la calidad ambiental en el medio construido de los asentamientos rurales. El cuantificador gráfico fue diseñado por Gómez (1997), Gómez (2005) y Velásquez (2003) y adaptado por la autora en el presente estudio.

\section{Fases de aplicación del instrumental práctico}

El instrumental práctico contiene las siguientes fases de aplicación para obtener la evaluación de la calidad ambiental del medio construido de los asentamientos rurales (ver figura 3 ):

- Información del asentamiento a través de la observación directa y aplicación de encuestas.

- Histogramas.

- Matriz síntesis.

- Expediente de mejoramiento de la calidad ambiental para el planeamiento y la gestión.

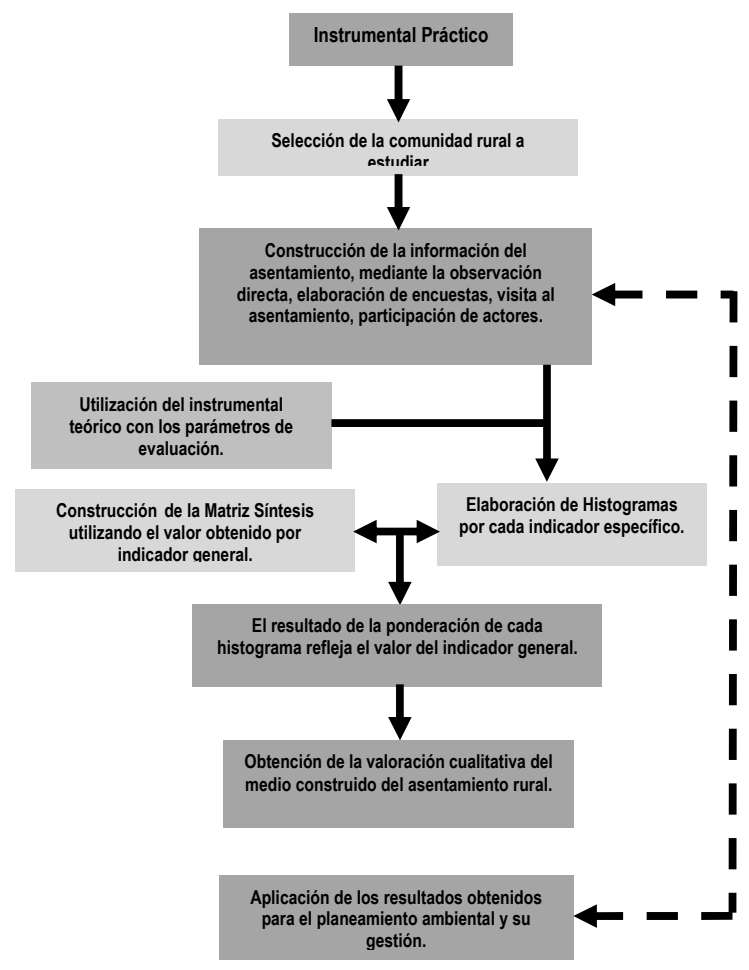

Fig. 3 Fases de aplicación del instrumental práctico.

Para la aplicación del instrumental fue necesario obtener la información de la situación actual del asentamiento objeto de estudio, la que debe proceder de la observación directa in situ, de encuestas y entrevistas a profundidad, de métodos participativos y del involucramiento de los actores implicados.

La tabla referenciada evidencia el comportamiento de cada indicador, previo conocimiento del objeto de estudio. Para obtener el valor de cada indicador, éste se somete a una ponderación (peso) y se le asigna la escala de valores: Alta importancia $=3$, Importancia $=$ 2 , Importancia baja $=1$. Este proceso resulta de las opiniones técnicas de los especialistas, de las entrevistas a los actores comunitarios y del criterio de la autora. 


\section{H. Hernández}

La ponderación no es absoluta para todos los asentamientos rurales, por lo que se recomienda que sea ajustada según las condiciones particulares de cada caso de estudio.

Con las evaluaciones de cada indicador referenciadas en los parámetros de evaluación (ver tabla 1), se obtienen los valores numéricos de la evaluación cualitativa. En los histogramas el valor cualitativo obtenido se complementa multiplicándolo por el peso de los indicadores, resultando el promedio de los valores cuantitativos de cada indicador, que al asociarse con los parámetros cualitativos permite obtener la valoración de la calidad ambiental (ver tabla 2).

Tabla 2 Histograma de Evaluación Ambiental.

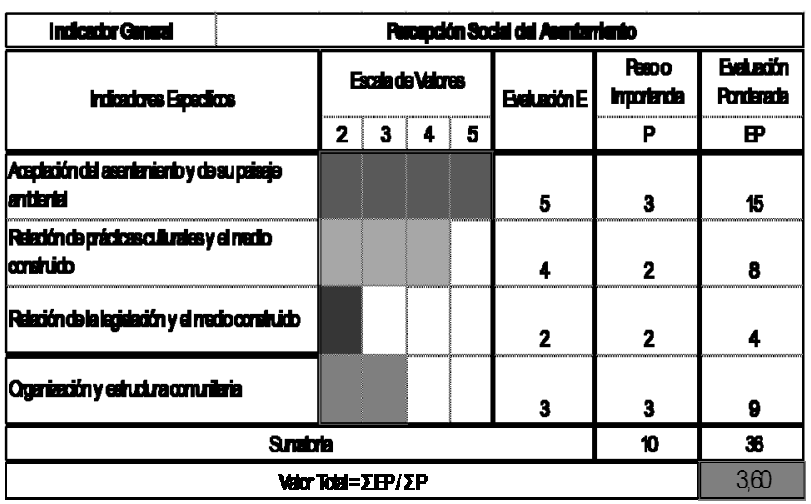

Este proceso se expresa por la siguiente fórmula matemática:

$\mathrm{E}=\Sigma \mathrm{EP} / \Sigma \mathrm{P}$

Donde para cada indicador:

$\mathrm{E}=$ es la evaluación

$\mathrm{P}=$ es el peso o importancia

$\mathrm{EP}=$ la evaluación ponderada

En el histograma se utilizan colores que facilitan la visualización de los resultados, siendo:

Verde oscuro: excelente

Amarillo: bueno

Anaranjado: regular

Rojo: malo

Los resultados de los histogramas permiten conocer el comportamiento de la calidad ambiental de cada asentamiento rural y posibilita determinar la problemática con especificidad, en donde el análisis integral del asentamiento se realiza con la matriz síntesis.
Esta matriz síntesis se obtiene del valor de cada uno de los histogramas, que establecen procesos estadísticos simples. La suma de cada histograma refleja el valor cualitativo y cuantitativo de cada Indicador General. Los valores de cada indicador general, sumados y promediados establecen el valor de la calidad ambiental del objeto de estudio (ver tabla 3).

Tabla 3 Matriz Síntesis de resultados.

\begin{tabular}{|c|c|c|c|c|c|}
\hline \multicolumn{6}{|c|}{ Asentam iento rural con arca Quebrada Honda } \\
\hline \multirow{2}{*}{ In dicador General } & \multicolumn{4}{|c|}{ Escala de Vabres } & \multirow{2}{*}{ Evaluación $E$} \\
\hline & 2 & 3 & 4 & 5 & \\
\hline Infraestrueturas Técnicas & & & & & 2.00 \\
\hline Patron de la Vivienda Rural & & & & & 3.00 \\
\hline Equipamiento Social y Especializado & & & & & 2.50 \\
\hline Percepcion Social I del Asentamie nto & & & & & 3.60 \\
\hline Integración del Asentemiento al entbmo & & & & & 4.00 \\
\hline Patrón Funcional del Asen tamiento & & & & & 3.86 \\
\hline Valorectba do ballad Anbten thi & & & $\overline{\mathbf{a r}}$ & & 3.16 \\
\hline
\end{tabular}

La matriz representa la síntesis de los resultados evaluativos, observándose con claridad cuáles indicadores generales presentan los problemas más significativos, contribuyendo a plantear y ejecutar soluciones encaminadas a equilibrar la deficiencia presentada.

\section{RESULTADOS Y DISCUSIÓN}

El instrumental resultante es un sistema de indicadores específicos y generales (ver tabla 4).

Tabla 4 Sistema de indicadores de calidad ambiental para un asentamiento rural.

\begin{tabular}{|c|c|}
\hline $\begin{array}{l}\text { Indicador } \\
\text { General (6) }\end{array}$ & Indicador Específico (15) \\
\hline \multirow{2}{*}{$\begin{array}{l}\text { Sistema de } \\
\text { Infraestructuras } \\
\text { Técnicas }\end{array}$} & $\begin{array}{l}\text { Existencia y calidad del sistema de infraestructura } \\
\text { técnica del asentamiento. }\end{array}$ \\
\hline & Consumo de energía continua. \\
\hline \multirow{2}{*}{$\begin{array}{l}\text { Características de } \\
\text { la vivienda rural }\end{array}$} & Elementos formales - espaciales de la vivienda rural. \\
\hline & Elementos funcionales - espaciales de la vivienda rural. \\
\hline \multirow{2}{*}{$\begin{array}{l}\text { Sistema de } \\
\text { Equipamiento }\end{array}$} & $\begin{array}{l}\text { Existencia y calidad del sistema de edificaciones del } \\
\text { asentamiento. }\end{array}$ \\
\hline & Existencia y calidad de los espacios públicos abiertos. \\
\hline \multirow{4}{*}{$\begin{array}{l}\text { Percepción social y } \\
\text { el asentamiento } \\
\text { rural }\end{array}$} & Aceptación del asentamiento y de su paisaje ambiental. \\
\hline & Relación de prácticas culturales y el medio construido. \\
\hline & Relación de la legislación y el medic \\
\hline & Organización y estructura comunitaria. \\
\hline \multirow{2}{*}{$\begin{array}{l}\text { El asentamiento } \\
\text { rural y su entorno } \\
\text { natural }\end{array}$} & $\begin{array}{l}\text { Relación entre los componentes físico - naturales y el } \\
\text { medio construido del asentamiento. }\end{array}$ \\
\hline & $\begin{array}{l}\text { Relación de los eventos naturales y el medio construido } \\
\text { del asentamiento. }\end{array}$ \\
\hline \multirow{3}{*}{$\begin{array}{l}\text { Accesibilidad del } \\
\text { asentamiento rural }\end{array}$} & Patrón funcional del asentamiento rural. \\
\hline & Sistema de producción local y acceso al empleo. \\
\hline & Seguridad social / ambiental del asentamiento rural. \\
\hline
\end{tabular}




\section{H. Hernández}

Con la finalidad de concluir el estudio del medio construido y la calidad ambiental, se elaboraró un expediente de cada uno de los asentamientos rurales evaluados, conteniendo la información actualizada en el proceso de aplicación instrumental.

Este expediente posibilitó elevar cualitativamente el manejo del asentamiento, una vez que quedan identificados los indicadores que afectan la calidad ambiental, puesto que le sirve a las instancias municipales, de ordenamiento territorial y de planificación física ambiental, para establecer objetivos y lineamientos de carácter sistémico e integral en el mejoramiento de los asentamientos rurales.

Con la realización de este expediente, se da inicio al proceso de la gestión ambiental, que comprende tres fases principales:

- Identificación de las afectaciones ambientales del medio construido.

- Jerarquización y prioridad de las afectaciones ambientales.

- Elaboración de los diferentes planes de acción por parte de los actores. Aquí se toma en cuenta la participación activa de los pobladores.

\section{CONCLUSIONES}

Se diseñó un instrumental que posibilita la evaluación de la calidad ambiental del medio construido de los asentamientos rurales, conformado por un instrumental teórico que engloba un sistema categorial y parámetros de evaluación cualitativos y cuantitativos, expresados en una tabla referenciada. Así como de un instrumental práctico establecido por histogramas y matriz síntesis.

Se determinaron los indicadores a través del Sistema de Interacciones, por contener la visión sistémica e integral del ambiente definido.

La obtención de los indicadores específicos se determinó por la asociación y significado de las variables interrelacionadas.

El proceso de evaluación se caracterizó por poseer un enfoque cualitativo, lo que permitió establecer las regularidades determinadas por un proceso de cuantificación de valores que sirvieron para caracterizar el sistema.
El instrumental teórico-práctico quedó conformado con la siguiente estructura y sistema categorial:

- Indicadores Específicos, Indicadores Generales, Parámetros Cualitativos y Escala de Evaluación.

- 6 Indicadores Generales, 15 Indicadores Específicos, obtenidos con una base de las 40 variables más significativas del sistema ambiental para el medio construido de los asentamientos rurales.

- En la tabla referenciada, la escala de evaluación es del 5 al 2.

- Parámetros cualitativos y cuantitativos en matrices e histogramas con rangos de: Excelente (verde oscuro, 4,50 - 5,00), Bueno (amarillo, 3,80 - 4,49), Regular (anaranjado, 3,00 - 3,79) y Malo (rojo, $2,00-2,99)$.

Este instrumental es el primer modelo en Nicaragua que mide la calidad ambiental del medio construido de los asentamientos rurales.

\section{REFERENCIAS}

Conesa F., \& Vitora, V. (1997). Guía Metodológica para la Evaluación del Impacto Ambiental. Editorial MP. Madrid, España

Delgado, P., \& Salcedo, T. Aspectos conceptuales sobre los indicadores de calidad de vida. Colombia. $\mathrm{S} / \mathrm{f}$.

Escobar, L. (2006). Indicadores sintéticos de calidad ambiental: un modelo general para grandes zonas urbanas. Eure. Agosto. Año / vol. XXXII, No 096. ISSN 0717-6236. Pontificia Universidad Católica de Chile. Santiago de Chile.

Gómez, G. (1997). Documento básico Curso Calidad de Vida. Especialidad en Vivienda Social y Calidad de Vida. UNI - PEAUT. Nicaragua.

Gómez, G. (2005). Instrumental para la evaluación de los componentes del medio construido que influyen en la calidad de vida en asentamientos rurales concentrados (ACR) de Santiago de Cuba. Tesis doctoral. Universidad de Oriente - ISPJAE. Santiago de Cuba.

Instituto Nacional de Información de Desarrollo INIDE (2007) Proyecto MECOVI - EMNV “Encuesta Nacional de Hogares sobre medición del nivel de vida 2005. Informe General." en: www.inide.gob.ni.2007. 


\section{H. Hernández}

Instituto Nicaragüense de Estadísticas y Censos INEC: (2007) “Censo de 1995, 2000, 2002, 2005." En: http://www. inec.gob.ni/

Instituto Nicaragüense de Estudios Territoriales/ Ministerio del Ambiente y los Recursos Naturales Documento Borrador 2003: Anteproyecto Ley General de Ordenamiento y Desarrollo Territorial de la República de Nicaragua. Nicaragua.

Mendoza, F. (2007). Sistema de Interacción para evaluar la calidad ambiental. Revista El Constructor. $\mathrm{N}^{\mathrm{o}} 19$. Nicaragua.

Ministerio del Ambiente y los Recursos Naturales MARENA, Segundo Proyecto de Desarrollo de Municipios Rurales (SPDMR) - Banco Central de Nicaragua (BCN) (2004) Indicadores Ambientales de Nicaragua. Volumen I. Nicaragua.

Pérez, A.. (2002). "La calidad de vida en los asentamientos rurales de Iberoamérica". Revista Geográfica Venezolana. ISSN 1012-1617, Vol. 43, No 1, (leído en 2006; http://dialnet.unirioja.es/servlet/revista)

Robles, T., \& Luna, R. (1999) Elaboración de indicadores para proyectos ambientales. PROARCA/CAPAS /AID.

Valentín, A., \& Spangenberg, J. (2000). “A guide to community sustainability indicators" en: environmental Impact Assessement.
Velásquez, L. (1998). Indicadores de calidad de vida de los asentamientos humanos. Red Latinoamericana de Estudios Ambientales Urbanos. Instituto de Estudios Urbanos de la Universidad Católica de Chile. Santiago de chile.

Velásquez, L. (1999) Propuesta de indicadores de calidad ambiental urbana. Red Latinoamericana de Estudios Ambientales Urbanos. Universidad del Medio Ambiente de Curitiba.

Velásquez, L. (2003). Propuesta de una metodología de planificación para el desarrollo urbano sostenible $y$ diseño de un sistema de evaluación de la sustentabilidad de ciudades medianas de América Latina. Tesis doctoral. Universitat Politècnica de Catalunya. Barcelona.

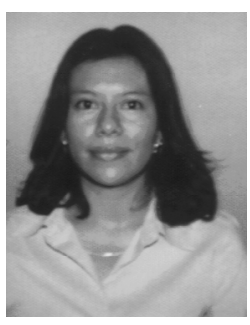

Heimdall Hernández se graduó de Arquitecto en la Universidad Nacional de Ingeniería (UNI) en 1996. Obtiene los grados científicos de Especialista en Vivienda Social y Calidad de Vida en 2001, Máster en Administración y Planificación de Proyectos Ambientales en 2007 y el grado de Doctor en Ciencias del Ambiente en 2008 en el Programa de Estudios Ambientales Urbano Territoriales de la UNI. Su área de investigación es la arquitectura y el ambiente con énfasis en los asentamientos rurales. Profesora Titular de la Universidad Nacional de Ingeniería. 